\title{
Optimization of Land Development for Single Axis Independent Row Trackers through Strip Cutting Method
}

\author{
Santanu Chatterjee, Sanjoy Mukherjee
}

\begin{abstract}
Single axis trackers (SAT) have always been of great importance and its discussions are becoming more and more apt for countries, like India, where the necessity of reaching at the lowest Levelized Cost of Energy (LCOE) targets are on though the lowest bids are lesser than Rs.2.5/Wp due to lowering of $P V$ module price. Since the requirement for lowering of $L C O E$ will claim low project cost as well as increment in generation thus singles axis trackers play a very important role. For lowering the cost of trackers, manufacturers are optimizing their design and making the trackers longer so that larger number of PV modules can be accommodated under one actuator or slew drive. This phenomenon attracts larger requirement for land leveling. Therefore, the prime objectives of this paper is to highlight how land leveling analysis through strip cutting method is better suited for SAT in comparison with conventional methods.
\end{abstract}

Keywords : Single Axis Tracker, Land Development, Cost optimization, Solar module mounting system.

\section{INTRODUCTION}

The importance of module mounting structure is to maximum generation in a PV plant by orienting the same towards sun in such a manner that the incident rays are perpendicular to the module surface. But since on 3D frame our earth has a motion not only in the XY plane but also in the $\mathrm{XZ}$ plane with a shift in the zenith angle, we need to consider the effect of both rotation and revolution to get the exact sun-path. Though both these changes occur daily, but the former is much more detectable as it happens every day whereas the latter is distributed throughout the year, getting detectable during the change of seasons [1].

So, trackers were developed as traditional mounting systems, both fixed tilted (FT) structures (i.e., facing towards south at a fixed tilt) and seasonally tilted fixed (STF) structures (i.e.; facing towards south with a fixed tilt with seasonal tilt adjustment facilities twice or thrice a year) had limitations of not having the capability of adjusting themselves as per the daily shift in sun-path.

But amongst single axis and dual axis trackers, the former became matter of prime importance as SAT-Horizontal is applicable all over India (especially South India) due to:

a) lower complexity of usage

b) lesser movable parts and thus incurring lesser operation and maintenance cost and replacements.

\section{BACKGROUND AND MOTIVATION}

Apart from increment in generation, few other observations need to be considered and they were:

1.Since trackers are highly location sensitive, for different location (higher and lower latitude areas) we should have different options of tracker. Thus there are block SAT and Independent Row SAT.

2. Trackers occupy higher space with respect to FT and STF structures of same DC capacity and

3. Trackers need more land leveling than other traditional structures as the different rows and strings are tied together to form a table which gets driven by a single actuator.

From the above discussion of our study, two prime aspects are right now in front of us where we wanted to work upon.

a) From above point no: 2 it can be understood that since trackers need more space for installation thus more area with respect to FT and STF is needed to be leveled as PV modules on each of tracker sits on one single torque tube.

b)From above Point no:3 we already can realize that block trackers will require more land leveling with respect to independent row tracker.

Therefore, we have only analyzed the land development requirement for independent row tracker as they are much more beneficial as per land shape adaptability as well as generation through independent backtracking, as shown in Fig.1 [2], in "Industry standard backtracking methodology".

In below mentioned Fig.1, the respective tracker manufacturer has shown how through independent adaptive backtracking the mutual shading issue will get avoided on East West side. But if we look into the fact considering a three dimensional view, then we shall be able to understand the effect of undulation on North-South side. If the land is undulated on the North-South side along with East-West side, the mixed effect will ensure more backtracking resulting in loss of generation for modules being not at optimum sun tracking angle. Thus land leveling is evident. 


\section{Optimization of Land Development for Single Axis Independent Row Trackers through Strip Cutting Method}

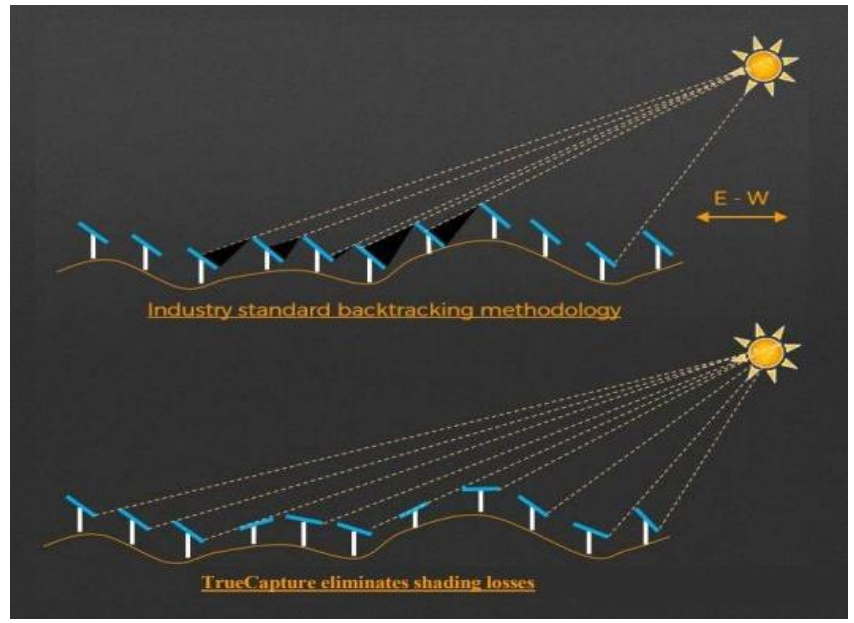

Fig. 1.Independent Row Trackers on undulated surface

\section{THE MODEL: BASIS OF ANALYSIS}

A full sized independent row single axis tracker table shall be of dimension 1.982 meters x 80.36 meters. It means that the tracker table shall be capable of holding 80 modules ( 4 strings) keeping all modules in landscape (single line) one after the other. This design is for $1000 \mathrm{~V}$ system where 20 numbers of modules shall be connected in a string.

Fig. 2 shows that the models of such full sized trackers are drawn on the natural contour level of a site. Site contour has been taken at an interval of 0.5 meters. Total 10 numbers of SATs (table numbers A to $\mathrm{J}$ ) are placed at a pitch distance of 7.00 meters. This pitch is the best possible pitch assumed as in majority of the sites, pitch distance for trackers could not be more than 5 to 5.5 meters (Indian context).

For single axis tracker we have assumed the allowable land slope along torque tube is $4 \%$ on the North-South side. Now as the land's natural profile is more than $4 \%$ hence we need to grade (land cutting) the site within the allowable limit of the SAT.

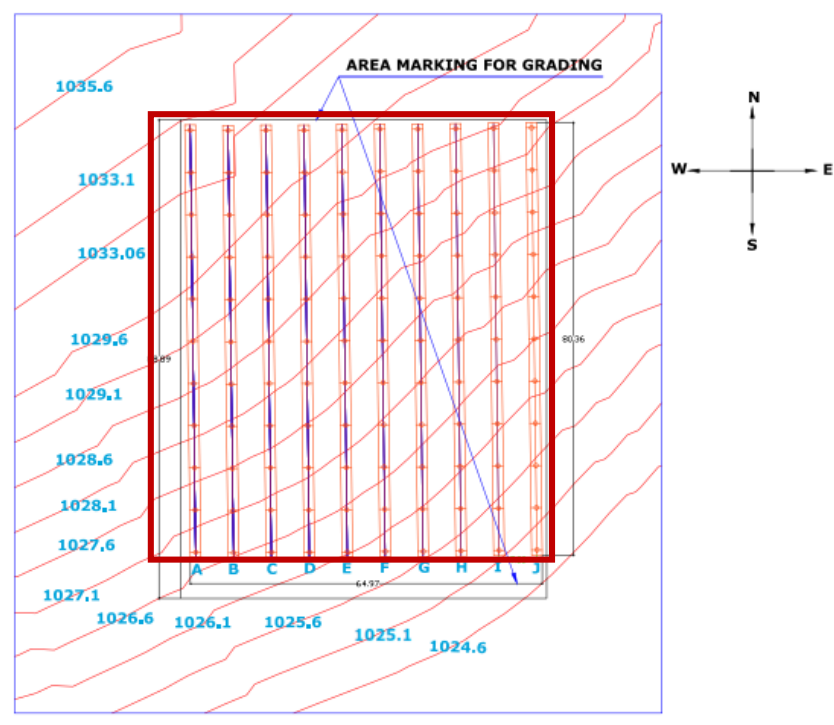

Fig. 2. Area Grading- Conventional Method

To be able to do that, as per conventional method of cutting technique (as shown in Fig.2), we can fix a grade level as per $4 \%$ slope $\&$ based on the natural profile we can cut the entire area (i.e. for all 10 numbers of tracker tables as a whole) so that finished ground level is equal to or less than allowable slope limit for the tracker, as shown in Fig.3.

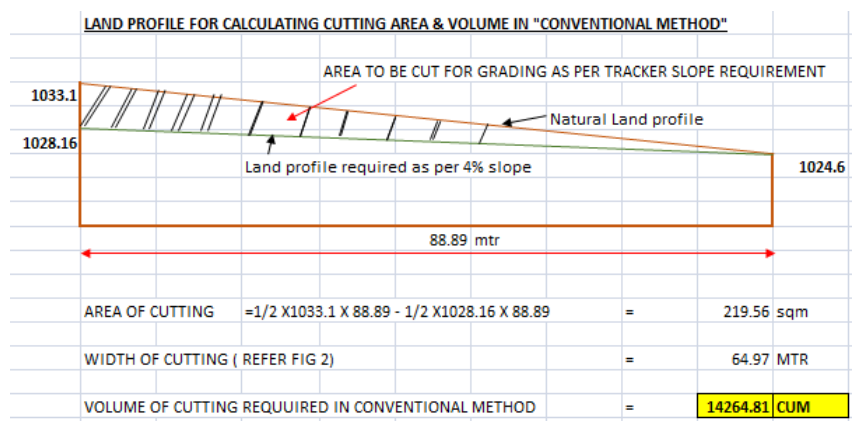

Fig. 3. Cutting volume- Conventional Method

The RED marked areas in Figure.2, Figure.4 \& Figure. 5 are the areas considered for cutting.
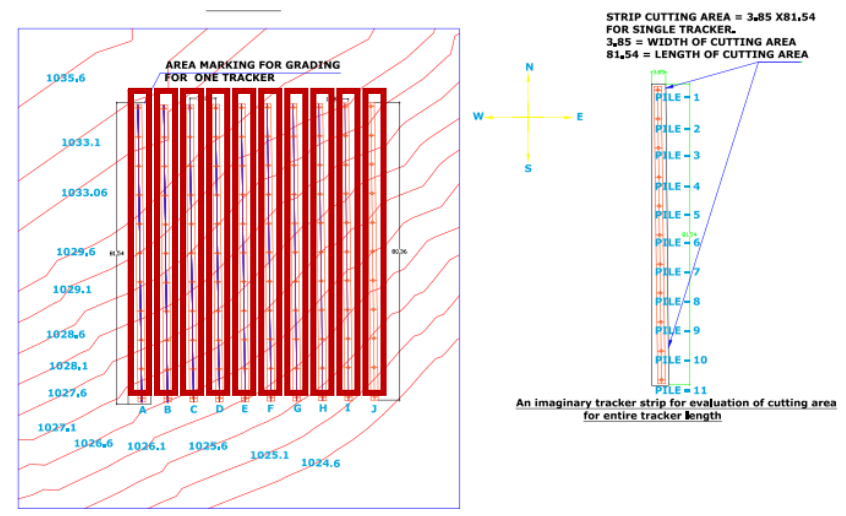

Fig. 4. Area Grading- Strip Cutting Method

On contrary to above mentioned conventional method of land cutting/ grading we can adopt strip cutting methods to calculate the land grading quantity. In this method instead of grading entire land, tracker table areas in the site are first subdivided into small rectangular strips (as shown in Fig.4). Typical dimension of the strip shall be the function of respective module $\&$ torque tube dimension. This concept is exactly similar to the philosophy of Finite Element Method (FEM), where to solve a large problem; it is subdivided into smaller, simpler parts, which are called finite elements. The simple equations that model these finite elements are then assembled into a larger system of equations that models the entire problem [3].

In this analysis, as shown in the below table (Table 1), we would check the spot level at each 11 nos. of pile positions of each and every independent row tracker in its entire length of 81.54 meters (as shown in Fig.4) but will grade the land considering the maximum and minimum heights, from north to south direction as we would never keep negative slope in case of trackers. From the above table (Table 1) it is clear that majority of the trackers are NOT at same plane.

Thus, from Table I, the total volume of cutting would be 3128.63 CUM (considering all 10 numbers of SATs) through Strip Cutting Method and the total volume of cutting is 
Table- I: Cutting volume- Strip Cutting Method

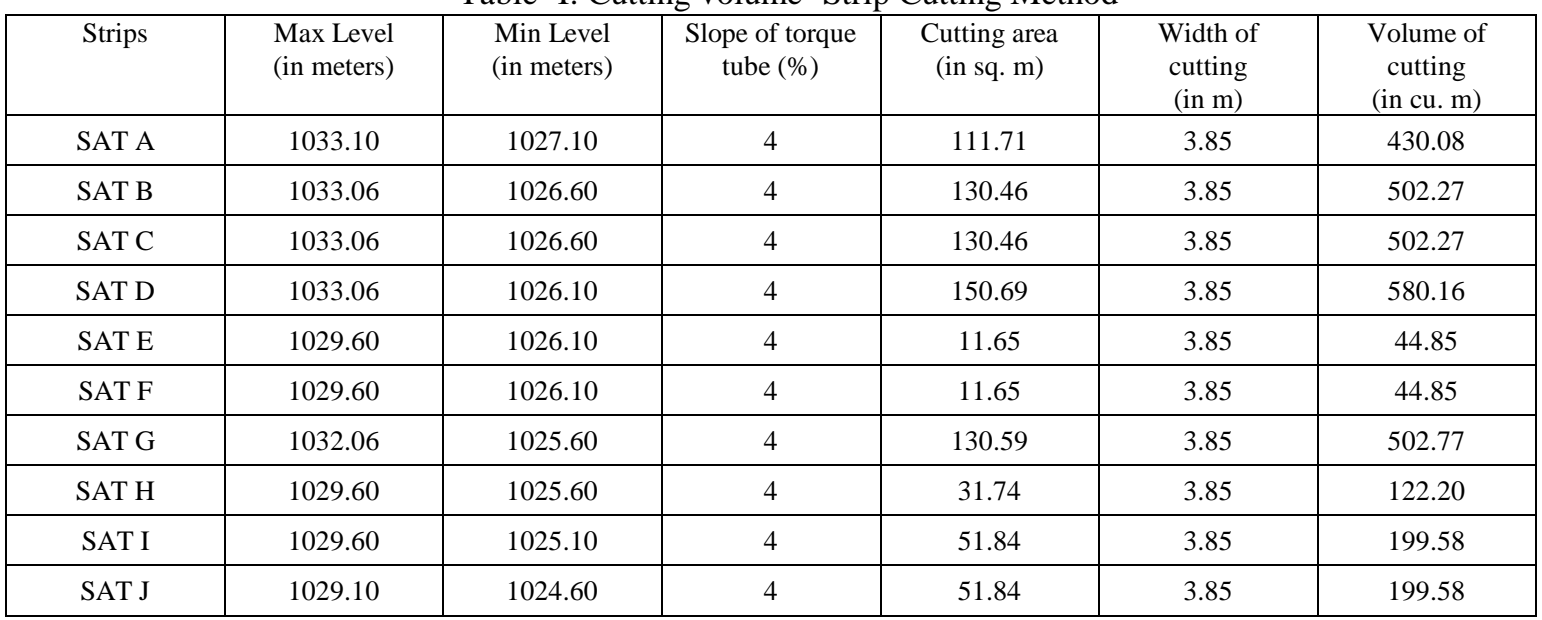

14264.81 CUM through Conventional Method.

As per below Fig.5, in certain situation it may be found that grading is not required for the entire tracker length/row. In such cases selective strip cutting can be performed accordingly to bring further optimization in terms of cutting volume.

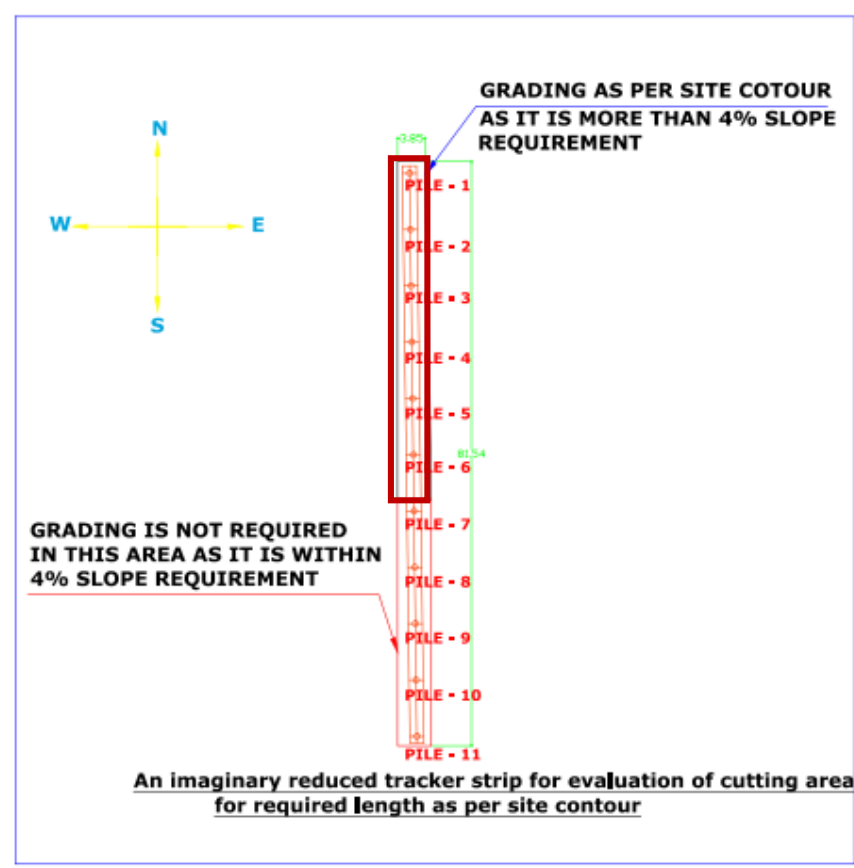

Fig. 5. Area Grading- Selective Strip Cutting

\section{CONCLUSION}

From the above analysis it is clear that volume of cutting through strip cutting method is just $\mathbf{2 1 . 9 3 \%}$ of the total volume as calculated through conventional method. Thus, it is confirmed that by strip cutting method it is possible to reduce volume of cutting as compared to conventional cutting, to a considerable scale. Hence for independent row SATs this method for land grading analysis will provide an optimized and economical result and help in making SAT viable in MW scale projects.

\section{WORK IN PROGRESS}

However, it may seem that the strip cutting analysis for land grading will be quite tedious in case of large solar sites (50 MW \& above). But actually, in case of large sites it is possible to demarcate land as per slope in north south direction into different zones and for each zone this type analysis can be carried out very easily through software. Thus, we are presently working into building the logics of this software which we will come up with shortly.

\section{REFERENCES}

1. International Science Index, Energy and Power Engineering Vol:4, No:4, 2017 waset.org/abstracts/59059

2. GTM \& NEXTracker Report https://www.nextracker.com/wp-content/uploads/2018/06/ GTM NEXTracker 6.21.18 Webinar_Slides_vsmFINALpptx.pd

3. https://en.wikipedia.org/wiki/Finite element method\#cite note--1

\section{AUTHORS PROFILE}

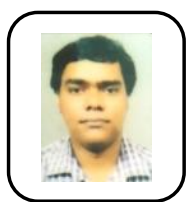

Santanu Chatterjee is a Bachelor of Engineering from Jadavpur University in Civil Engineering presently working in the field of civil design for transmission lines and substation in RGM International (India) Pvt. Ltd. He has been also involved in civil design of several solar power plants. He also takes interest in concepts of string theory and quantum mechanics and recently got his paper selected for International Conference on Mathematical Modeling in Physical Sciences and one of his papers got awarded as best scientific paper at NIT Durgapur, India. Presently he is working on development of a calculation tool for transmission line that would enable engineers to estimate volume of civil works in a very less time.

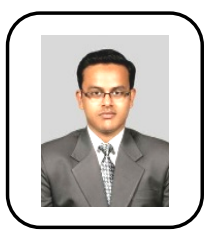

Sanjoy Mukherjee is a Bachelor of Technology from Techno India in Electronics and is a former researcher at Jadavpur University at Department of Architecture on Energy Efficient Built Environment. His areas of work include R\&D on solar tracking mechanism, energy storage and inverters. He has published several papers on renewable energy at European Union PV Solar Energy Conference (Germany), Internationa conference of Illumination (India), International conference on Clean and Renewable Energy (India) as well as co-authored papers at IIT Roorkee and other eminent conferences. He was awarded as Best Engineering Employee by Vikram Solar in 2015 and one of his papers also got awarded as best scientific paper at NIT Durgapur, India.

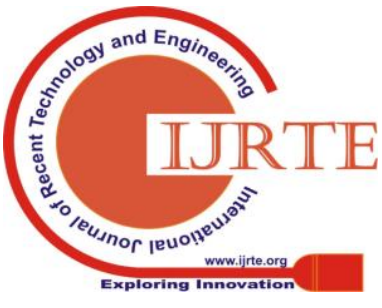

